\title{
Chest wall trauma leading to a metallic foreign body in the right subclavian vein: a case report
}

\author{
Anping Chen ${ }^{1}$, Gang $\mathrm{Xu}^{1}$, Qingyong Cai ${ }^{1}$, Yongxiang Song ${ }^{1}$, Kurt Ruetzler ${ }^{2}$, Robert E. Merritt ${ }^{3}$, \\ Cheng Chen ${ }^{1}$
}

${ }^{1}$ Department of Thoracic Surgery, Affiliated Hospital of Zunyi Medical University, Zunyi, China; ${ }^{2}$ Departments of Outcomes Research and General Anesthesiology, Cleveland Clinic, Main Campus, Cleveland, OH, USA; ${ }^{3}$ Division of Thoracic Surgery, Department of Surgery, The Ohio State University Wexner Medical Center, Columbus, OH, USA

Correspondence to: Cheng Chen. Department of Thoracic Surgery, Affiliated Hospital of Zunyi Medical University, No. 149 Dalian Road, Zunyi 563000, China. Email: 375647557@qq.com.

Submitted Nov 03, 2020. Accepted for publication Jan 30, 2021.

doi: $10.21037 /$ jtd-21-68

View this article at: http://dx.doi.org/10.21037/jtd-21-68

\section{Introduction}

Chest trauma is common in thoracic surgery inpatients and one of the main causes of death due to trauma (1). If the treatment of chest trauma is not timely, death due to pneumothorax, hemothorax, hemopneumothorax and other injuries may occur (1). In this case report, a renovation worker was admitted to hospital for breaking the tip of the rotating head of the machine during work, which shot into his chest wall and remained there for 1 hour. The initial diagnosis was a residual foreign body in the chest wall, and skin damage was observed under the right clavicle, with only minor bleeding, pain, and other symptoms being noted. We present the following article in accordance with the CARE reporting checklist (available at http://dx.doi.org/10.21037/ jtd-21-68).

\section{Case presentation}

The patient was a 24 -year-old male renovation worker. One hour before admission, he suffered mechanical damage due to carelessness, which caused injury to his right chest wall. After emergency dressing in the out-of-hospital setting, the patient was admitted to our hospital. Upon admission, physical examination showed skin laceration with a diameter of about $0.5 \mathrm{~cm}$ below the midpoint of the right clavicle and a small amount of bleeding (Figure 1). The patient had no symptoms of shock, local pain, swelling, right upper limb dysfunction, chest tightness, shortness of breath, or respiratory dysfunction. Chest X-ray and chest computed tomography (CT) examination were performed, and a high-density shadow was found below the clavicle of the right chest wall, without pneumothorax. Enhanced CT (Figure 2) revealed a metallic foreign body present between the right subclavian artery and vein; CT angiography indicated (Figure 3) that the foreign body could have been in the subclavian vein. Considering that the foreign body might have been located around or inside the blood vessels, which could have easily led to blood vessel rupture and bleeding, foreign body migration, thrombosis, etc., the foreign body was removed under local anesthesia. Intraoperative extension of the wound in combination with intraoperative radiography was performed to determine the degree of foreign body injury to the blood vessels (Figure 4). The presence of a foreign body in the right subclavian vein was considered. The patient after anesthesia the incision was extended to $10 \mathrm{~cm}$ along the subclavian bone, the soft tissue was fully exposed, the right subclavian vein was exposed, and side wall damage and bleeding became visible. After adequate exposure of the subclavian vein, the vessel was clamped proximally and distally of the foreign body, and cut lengthwise. A $1 \mathrm{~cm} \times 0.5 \mathrm{~cm} \times 0.5 \mathrm{~cm}$ metal foreign body and a small thrombus were found (Figures 5 and 6 ). The foreign body was entirely removed and the vessel was sutured lengthwise. The patient was discharged 4 days after surgery, with forearm suspension, and anti-infection and anticoagulation therapy had been given. No complications such as bacteremia, thrombus and infection occurred during perioperative period; The patient is quite satisfied with the 


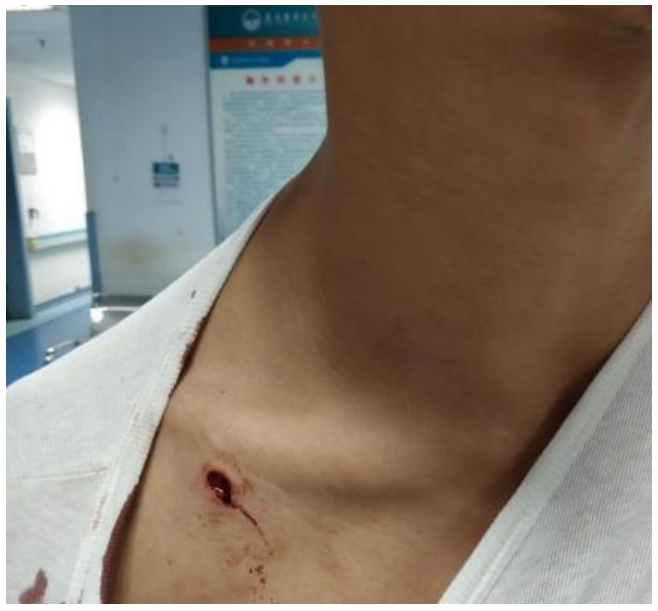

Figure 1 A skin laceration about $0.5 \mathrm{~cm}$ below the midpoint of the right clavicle with a small amount of bleeding was observed preoperatively.

diagnosis and treatment process;

All procedures performed in studies involving human participants were in accordance with the ethical standards of the institutional and/or national research committee(s) and with the Helsinki Declaration (as revised in 2013). Written informed consent was obtained from the patient for publication of this study and any accompanying images.

\section{iMDT discussion}

\section{Discussion among physicians from Affiliated Hospital of Zunyi Medical University}

An in vivo foreign body is a common surgical emergency. Usually the cause of such an injury is traumatic or iatrogenic. The pathogenesis of traumatic injury is complex and can thus be easily misdiagnosed or missed. Chest wallpenetrating injury often causes multiple injuries to the chest wall structures, including the skin, muscle, blood vessels, ribs, etc., and in the case of serious life-threatening injuries, emergency surgical intervention is required. The patient or the doctor may easily find a large foreign body outside the chest wall, but for a foreign body located in the gap between the soft tissues in the body, there may be only skin and soft tissue damage on the outside, and serious vascular, nerve, bone, or other injuries on the inside, resulting in missed diagnosis. Some foreign bodies remaining in the interstitial space can move with muscle contractions, while some remaining in the blood vessels may then enter the heart
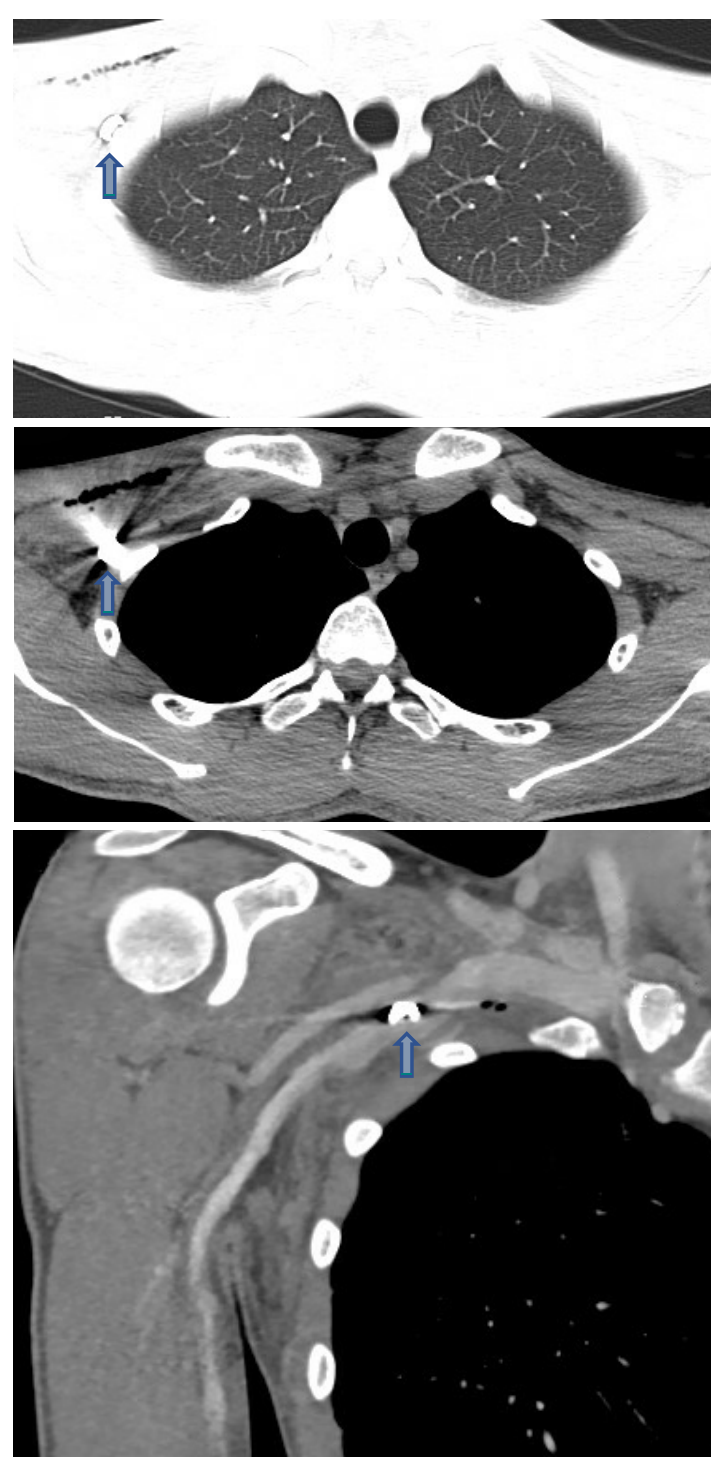

Figure 2 A high-density shadow was observed on the coronary position of chest CT between the right subclavian artery and vein. CT, computed tomography.

and large blood vessels through blood circulation, causing serious injuries and complications such as embolism, infection, heart injury, massive bleeding, and even death.

\section{Department of General Surgery}

Common intravascular foreign bodies are iatrogenic injuries, and the main foreign bodies include needles (2), intravenous intubation (3), guidewires (4), stents (5), and inferior vena cava filter stents (6). Different foreign bodies in blood vessels may show different symptoms according 


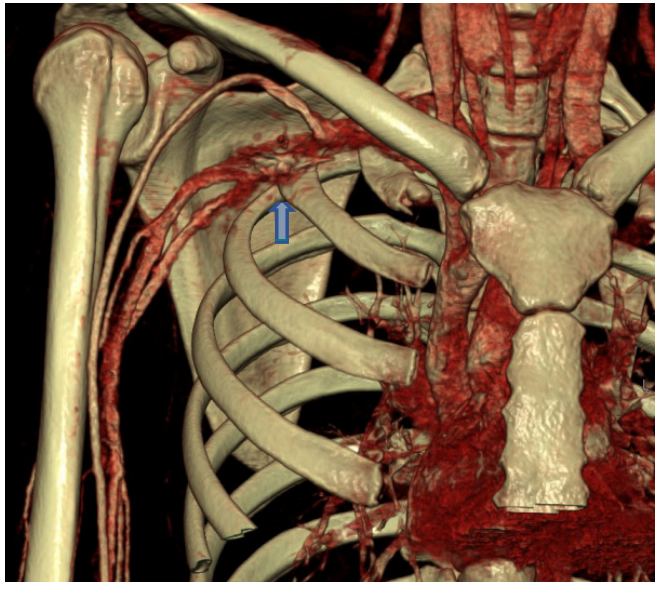

Figure 3 Chest CT angiography and 3D reconstruction showed foreign bodies in the subclavian vein. CT, computed tomography.

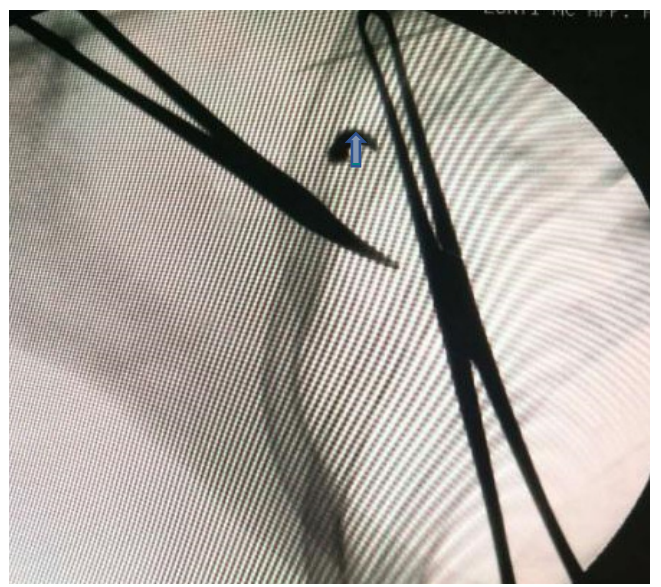

Figure 4 Intraoperative c-arm radiography confirmed the presence of a foreign body in the right subclavian vein area.

to the location in the body. However, local pain and discomfort are the most common clinical manifestations in symptomatic foreign body patients. Trauma may lead to the residual foreign body entering the blood vessels. Due to the rapid onset and rapid progress, most symptoms in such cases are severe and can include pain and shock caused by blood loss and limb dysfunction.

\section{Department of Vascular Surgery}

The diagnosis of an in vivo foreign body is relatively simple and involves clear pathogenesis and physical examination. If a residual foreign body is suspected, common methods such as X-ray, CT scan, and angiography can be used for

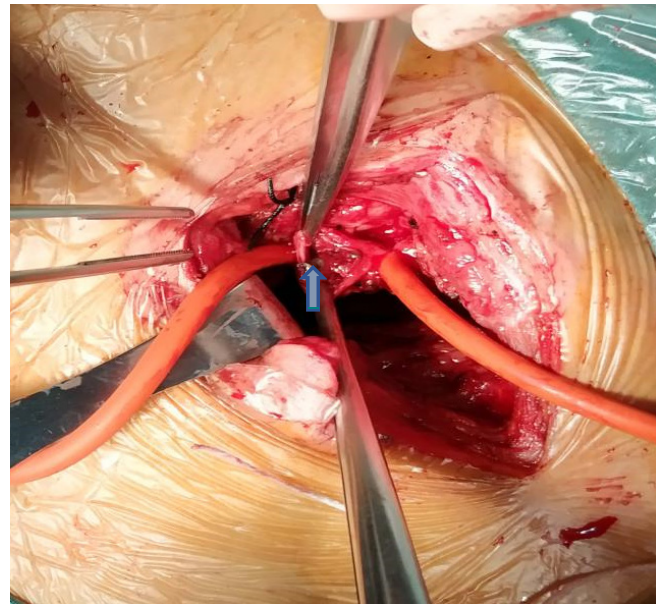

Figure 5 Metallic foreign bodies (arrow) were seen in the vein, and a small amount of thrombosis was observed in the lumen.

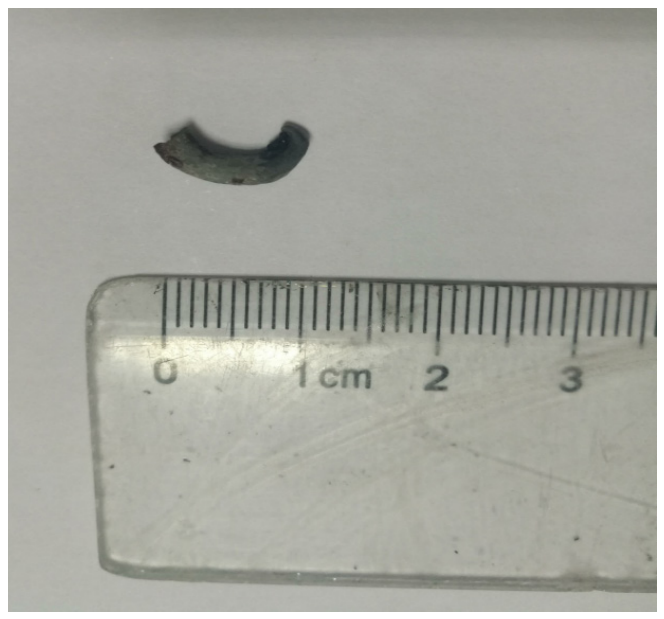

Figure 6 A long metallic foreign body, $1 \mathrm{~cm} \times 0.5 \mathrm{~cm} \times 0.5 \mathrm{~cm}$ in size.

diagnosis. However, the effectiveness of foreign body diagnosis depends on the nature and density of the foreign body. High-density and low-density foreign bodies have different manifestations in imaging examination, so different methods should be selected for examination. Some foreign bodies cannot be confirmed by the above methods, and can only be confirmed by direct surgery or vascular intervention.

The main purpose of surgical treatment is to save lives, remove foreign bodies, restore function, and reduce related complications. It is important to determine whether the residual foreign body in the chest wall needs to be removed 
by surgery, whether the it manifests symptoms in the soft tissue, whether it affects bodily functions, and whether it poses potential threat to the surrounding organs. The patient and other social factors should also be considered. However, for those foreign bodies that are mobile, it is often difficult to evaluate the distance moved, and dynamic $\mathrm{X}$-ray fluoroscopy is needed to determine the positioning. Therefore, residual large foreign bodies or foreign bodies present around the blood vessels and nerves may pose a serious threat and involve serious complications.

\section{Several issues regarding to the diagnosis and treatment of this patient for further discussion}

Question 1. What is the best time to remove the foreign body in the chest wall? What if this optimal time is missed? How should residual foreign bodies be managed? How to deal with residual foreign body?

\section{Expert opinion 1: Dr. Kurt Ruetzler}

Emergency treatment of patients with penetrating injuries depends on cardiovascular stability of the injured patient. Clinicians should strive for immediate clinical assessment and subsequent triage and surgical removal of foreign bodies. Imaging evaluation depends on patient's conditions and suspected injuries. Most penetrating injuries involve only the chest wall and lung parenchyma and may initially be imagined with chest radiography. Entry and exit wounds should be demarcated by radiopaque markers to help approximate the injury trajectory and its proximity to the mediastinum. Imaging evaluation of patients with suspected mediastinal or cardiac injury depends mostly on the patient's conditions, as these injuries are usually accompanied by severe blood loss and shock symptoms. Focused assessment by sonography in trauma (FAST) should be used to initially assess a potential penetrating mediastinal injury and evaluate any pericardial fluid. A subsequent CT angiography (CTA) should be performed afterwards.

\section{Expert opinion 2: Dr. Robert E. Merritt}

Foreign bodies should be removed from the chest wall upon presentation to minimize the risk of infection. Metallic foreign bodies located in the chest wall can migrate into the pleural space and result in vascular or lung injury as well if not removed promptly. If chest wall foreign bodies can't be removed promptly, then the foreign body should be surgical removed when possible. Blunt shaped foreign bodies can be left in placed if they cannot be removed safely with minimal surrounding tissue damage.

Question 2. What should be done if a foreign body enters the blood circulation through a blood vessel? If foreign body enters blood circulation through blood vessel, bow should deal with?

\section{Expert opinion 1: Dr. Kurt Ruetzler}

If the foreign body is confirmed to remain in subcutaneous structures without injuries of any major vessels or nerves, surgical removal under local anesthesia should be considered. If major structures like vessels, nerves or bones are injured, general anesthesia and close hemodynamic monitoring should be considered. Subsequent transfer to ICU should be considered after major vascular injury or blood loss.

\section{Expert opinion 2: Dr. Robert E. Merritt}

Foreign bodies that enter the blood circulation trough blood vessels should be removed immediately due to the risk of end organ ischemia. The foreign body that enters the blood circulation can be removed with endovascular retrieval. The foreign body that enters the blood circulation can be removed by direct surgical extraction. The blood vessel is isolated with vascular clamps and the blood vessel is incised. The foreign body is then extracted and the blood vessel is closed.

\section{Question 3. What are the difficulties involved in removing a foreign body from the chest wall?}

\section{Expert opinion 1: Dr. Kurt Ruetzler}

Postoperatively, even in asymptomatic patients, a chest radiography follow-up should be performed after 3-6 hours to rule out the development of delayed pneumothorax, though current evidence suggests that this interval should not exceed 1 hour.

\section{Expert opinion 2: Dr. Robert E. Merritt}

Foreign bodies that are removed in a delayed fashion may become incorporated in the soft tissues of the chest wall and may be difficult to removed. In addition, chest wall foreign bodies could migrate into the pleural cavity, which required thoracoscopy or thoracotomy for removal. Finally, foreign bodies in the chest wall may also migrated into blood vessels which is a serious complication and requires immediate endovascular or surgical intervention. 


\section{Conclusions}

We report the case of posttraumatic endovascular foreign body retention. The trauma was clearly defined on the right chest wall, skin damage was visible under the right clavicle, and only bleeding and pain symptoms were observed, with no chest distress, dyspnea, shock, or other symptoms. Auxiliary examination was conducted to confirm the residual foreign body, and no hemothorax, pneumothorax, fracture, or other conditions were encountered. An intraoperative foreign body was found in the right subclavian vein lumen; it did not flow into the heart with the blood or cause serious complications, which is very rare.

For chest wall foreign bodies, we believe that treatment should be determined according to the size, type and location of the foreign body, such as small foreign body, no risk of bleeding and dysfunction, consider non-surgical treatment; such as adjacent blood vessels, pleural cavity, articular cavity, easy to cause bleeding, hemopneumothorax, joint movement disorders, recommend active surgical treatment; when foreign bodies enter the blood circulation, such as into the left heart system, foreign bodies can follow the blood circulation into the systemic trachea, may cause organ ischemia infarction; whether myocardial infarction, the probability of occurrence should be relatively low; if the foreign body enters the right heart system, it may enter the pulmonary circulation with the blood circulation, may further produce thrombus or foreign body clogging the pulmonary artery and lead to pulmonary embolism, in case of emergency, surgical treatment is recommended.

\section{Acknowledgments}

Funding: None.

\section{Footnote}

Reporting Checklist: The authors have completed the CARE reporting checklist. Available at http://dx.doi.org/10.21037/ jtd-21-68

Conflicts of Interest: All authors have completed the ICMJE uniform disclosure form (available at http://dx.doi. org/10.21037/jtd-21-68). Robert E. Merritt reports speaker fees from Intuitive Surgical, outside the submitted work. The other authors have no conflicts of interest to declare. Ethical Statement: The authors are accountable for all aspects of the work in ensuring that questions related to the accuracy or integrity of any part of the work are appropriately investigated and resolved. All procedures performed in studies involving human participants were in accordance with the ethical standards of the institutional and/or national research committee(s) and with the Helsinki Declaration (as revised in 2013). Written informed consent was obtained from the patient for publication of this study and any accompanying images.

Open Access Statement: This is an Open Access article distributed in accordance with the Creative Commons Attribution-NonCommercial-NoDerivs 4.0 International License (CC BY-NC-ND 4.0), which permits the noncommercial replication and distribution of the article with the strict proviso that no changes or edits are made and the original work is properly cited (including links to both the formal publication through the relevant DOI and the license). See: https://creativecommons.org/licenses/by-nc-nd/4.0/.

\section{References}

1. Tekinbaş C, Eroğlu A, Kürkçüoğlu IC, et al. Chest trauma: analysis of 592 cases. Ulus Travma Acil Cerrahi Derg 2003;9:275-80.

2. Low GS, Jenkins NP, Prendergast BD. Images in cardiology. Needle embolism in an intravenous drug user. Heart 2006;92:315.

3. Singh A, Kaur A, Singh M, et al. CT Guided Removal of Iatrogenic Foreign Body: A Broken Intravenous Cannula. J Clin Diagn Res 2015;9:PD28-9.

4. Cat BG, Guler S, Soyuduru M, et al. Complete guidewire retention after femoral vein catheterization. Ann Saudi Med 2015;35:479-81.

5. Ho JM, Kahan J, Supariwala A, et al. Vascular stent fracture and migration to pulmonary artery during arteriovenous shunt thrombectomy. J Vasc Access 2013;14:175-9.

6. Shennib H, Bowles B, Hickle K. Migration of a fractured inferior vena cava filter strut to the right ventricle of the heart: a case report. J Cardiothorac Surg 2014;9:183.

Cite this article as: Chen A, Xu G, Cai Q, Song Y, Ruetzler $\mathrm{K}$, Merritt RE, Chen C. Chest wall trauma leading to a metallic foreign body in the right subclavian vein: a case report. J Thorac Dis 2021;13(2):1286-1290. doi: 10.21037/jtd-21-68 